\title{
Epidemiology Characteristics and Trends of Incidence and Morphology of Stomach Cancer in Iran
}

\author{
Zeinab Almasi ${ }^{1}$, Hosein Rafiemanesh ${ }^{2}$, Hamid Salehiniya ${ }^{2,3 *}$
}

\begin{abstract}
Background: Stomach cancer is the fourth most common cancer and the second leading cause of cancerrelated death through the world. It is predicted that the number of new cancer cases will be more than 15 million cases by 2020 . Regarding the lack of studies on this topic in the country, we have thoroughly examined the patho-epidemiology of stomach cancer in Iran. Materials and Methods: In this cross- sectional study data were collected retrospectively reviewing all new stomach cancer patients in Cancer Registry Center report of health deputy for Iran during a 6-year period (2003-2008). The study also examined the morphology of common stomach cancers. Trends in incidence and morphology underwent joinpoint regression analysis. Results: During the six-year period, a total of 35,171 cases of stomach cancer were registered. Average age standardized rate for females and males were equal to 7.1 and 15.1 per 100,000 persons, respectively. Most common histological type was adenocarcinoma, NOS with 21,980 cases $(62.50 \%)$. The annual percentage change (APC) in age-standardized incidence rate (per 100,000) was increase in both females and males at 11.1 (CI: 4.3 to 18.3) and 9.2 (CI: 5.2 to 13.4), respectively. Conclusions: According to our results, the incidence of gastric cancer is increasing in Iran, so further epidemiological studies into the etiology and early detection are essential.
\end{abstract}

Keywords: Stomach cancer - trend - incidence - morphology - Iran

Asian Pac J Cancer Prev, 16 (7), 2757-2761

\section{Introduction}

Cancer is the second most common cause of death among developed countries after cardiovascular disease and is the third common cause of mortality in developing countries (Bener et al., 2008). It is predicted that the number of new cancer cases give rise to more than 15 million cases and their related deaths will be increased to 12 million deaths by 2020 (Han et al., 2011). Stomach cancer is the fourth most common cancer and the second leading cause of cancer-related death through the world.

Classification of stomach cancer is based on two factors i.e. the anatomic appearance of cancer (cardia and non-cardia) and histological type of disease (enteric and non-enteric). Each of these kinds is different from the etiology, symptoms and prognosis point of view. The main cause of non-cardia cancer in the world is Helicobacter pylori. Today, Cardia cancer incidence is rising but non-Cardia cancer incidence is falling. Also enteric form has better prognosis than diffuse type (Kim, 2008). According to recent statistics published by WHO, stomach cancer is responsible for about $10.4 \%$ of death toll and each year approximately 930,000 new cases of cancer in the world are recognized which at least 700,000 of them die because of this disease. Overall, the incidence and mortality of stomach cancer has declined dramatically in the past 70 years (Shafaghi et al., 2012). This cancer is the most common cause of cancer-attributed deaths in Iran (Derakhshan et al., 2004)and the most common type of cancer in the North and North West of the country

Comparison between cardia cancer cases in Ardabil with other parts of the world shows that the incidence of Cardia cancer in Ardabil has the highest rate in the world, even higher than North America, Western Europe, Japan and Korea (Babaei et al., 2005).

Unlike the Western Europe, North America, and Japan, the incidence of stomach cancer has been rising over the past 30 years in Iran particularly in Azerbaijan, (especially in Ardabil province) which has the highest rate (Yazdizadeh et al., 2005; Abdi-Rad et al., 2006; Kim, 2008).

The highest age-specific rate (ASR) for stomach cancer in Ardabil province is equal to ASR=49/1 (for men) and $\mathrm{ASR}=25 / 4$ for women(Babaei et al., 2005; Kim, 2008). In all areas of Iran, non-Cardia cancer with age-standardized rate equal to 12 is considred as the average rate in the country (Babaei et al., 2005; Yazdizadeh et al., 2005). At the southern part of Iran, non-Cardia cases constitute a large part of the stomach cancer (Ahari et al., 2013) .Incidence of disease increases with age. The highest incidence is attributed to age group of 50-70 years old (Moore et al., 2010). 
Helicobacter pylorus is the most important etiology for stomach cancer. Stomach cancer is a multi-factorial disease and it has been known that some factors such as geographical diversity, time trend, the environment, migration and lifestyle are among the basic factors which are effective on stomach cancer incidence. The risk factors for disease include Helicobacter pylori infection, diet, smoking, obesity and geographic areas which gastro esophageal reflux is common there (Babaei et al., 2009).

Due to the increasing trend of disease incidence, high fatality rate and adverse consequences which is related to disease and the way of response to treatment, so the study and understanding this cancer and determining it's pattern is very important and considered to be as the first step for the planning and coordination for national control of the disease. Regarding the lack of studies on this topic in the country, we have thoroughly examined the pathoepidemiology of stomach cancer in Iran.

\section{Materials and Methods}

\section{Data source}

This is cross-sectional study in Iran. The data were collected from Cancer Registry Center report of health deputy which is based on Iran ministry of health guideline(Goya, 2007). Data were collected retrospectively reviewing all new stomach cancer patients in cancer registry center report of health deputy for Iran during a 6-year period (2003-2008). stomach cancer was defined as icd-o c16 (Fritz, 2000). This study survey adenocarcinoma, nos (8140/3), signet ring cell carcinoma $(8490 / 3)$, adenocarcinoma, intestinal type $(8144 / 3)$, carcinoma, diffuse type (8145/3), carcinoma, nos (8010/3), mucinous adenocarcinoma (8480/3) and mucin-producing adenocarcinoma (8481/3) morphology.

\section{Statistical analysis}

Age-standardized rates of cancer incidence were calculated by the direct standardization method, using the world standard population as a reference. To describe incidence time trends, we carried out joinpoint regression analysis using the software Joinpoint Regression Program, Version 4.1.1.1 October 2014. As well to evaluate the morphological changes, were obtained the percentage allocated for kind of morphological types. So to analysis morphology change percentage trends for six year, we carried out joinpoint regression analysis using the software Joinpoint Regression Program. The analysis included logarithmic transformation of the rates, standard error, maximum number of one joinpoints, and minimum of six years between zero joinpoints. All other program parameters were set to default values. The aim of the approach is to identify possible joinpoints where a significant change in the trend occurs. In this study 0 joinpiont (Full model) was a significant model. The final model selected was the most parsimonious of these, with the estimated annual percent change (APC) based on the trend within each segment. In describing trends, the terms "significant increase" or "significant decrease" signify that the slope of the trend was statistically significant $(\mathrm{p}<0.05)$. All statistical tests were two sided.

\section{Results}

During a six-year study, totally 35,171 cases of stomach cancer were registered, of which 9920 cases $(28.20 \%)$ were female and 25251 cases $(71.80 \%)$ were male. The sex ratio (male to female) for disease was equal to 2.54. Average age standardized rate for female and male group were equal to $7.06(\mathrm{SD}=1.60)$ and $15.06(\mathrm{SD}=2.57)$ per hundred thousand persons, respectively. Four common histological types include ADENOCARCINOMA, NOS with 21,980 cases $(62.50 \%)$, SIGNET RING CELL CARCINOMA with 3856 cases $(10.96 \%)$, ADENOCARCINOMA, INTESTINALTYPE with 3677 cases $(10.45 \%)$ and CARCINOMA, DIFFUSE TYPE with 1467 cases $(4.17 \%)$. Overall, $88.10 \%$ of stomach cancer cases were related to the four above mentioned histological types.

\section{Epidemiological trend}

Age-standardized incidence rate (per 100,000) for disease among women was increased from 5.20 to 10 and from 11.37 to 19.16 among men during 2003 to 2008 (Table 1).

Joinpoint Trend Analysis showed a significant increase for age-standardized incidence rate (ASIR) for both sexes. The annual percentage change (APC) of standardized incidence rate for females and males were 11.1 (CI: 4.3 to 18.3) and 9.2 (CI: 5.2 to 13.4), respectively (Figure 1).

\section{Morphological process}

During the study years, allocated percentages for histology types were different (Table 2).

Table 1. Frequency, Crude and Standardized Incidence of Stomach Cancer by Sex, During the Years 2003 to 2008

\begin{tabular}{lcccccccc}
\hline Year & \multicolumn{3}{c}{ Female } & & \multicolumn{3}{c}{ Male } & \\
\cline { 6 - 7 } & N. & CIR & ASIR & & N. & CIR & ASIR & M:F \\
\hline 2003 & 1166 & 3.6 & 5.2 & 3088 & 9.05 & 11.37 & 2.65 \\
2004 & 1439 & 4.37 & 6.42 & & 3770 & 10.87 & 13.74 & 2.62 \\
2005 & 1624 & 4.73 & 6.74 & & 4212 & 11.65 & 14.9 & 2.59 \\
2006 & 1603 & 4.69 & 6.65 & & 4299 & 11.94 & 15.24 & 2.68 \\
2007 & 1735 & 5.08 & 7.38 & & 4484 & 12.46 & 15.93 & 2.58 \\
2008 & 2353 & 6.89 & 10.00 & 5398 & 14.99 & 19.16 & 2.29 \\
\hline
\end{tabular}

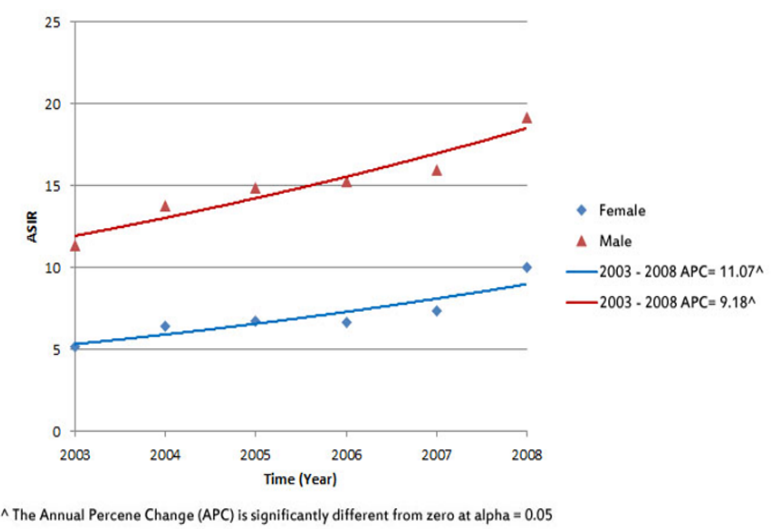

Figure 1. Joinpoint Analysis for Incidence of Stomach Cancer (Male and Female) in Iran, 2004-2009; ASRAge-standardized rate per 100000 (Using World Standard Population) 
Table 2. Frequency and Percent Change in the Percentage Allocated to the Seven Morphology Data for Stomach Cancer (2008-2003)

\begin{tabular}{|c|c|c|c|c|c|c|c|c|c|c|c|c|}
\hline & \multicolumn{6}{|c|}{ Count } & \multicolumn{6}{|c|}{ Percent } \\
\hline & 2003 & 2004 & 2005 & 2006 & 2007 & 2008 & 2003 & 2004 & 2005 & 2006 & 2007 & 2008 \\
\hline \multicolumn{13}{|l|}{ Female } \\
\hline Adenocarc & 730 & 944 & 988 & 887 & 973 & 1386 & 62.61 & 65.60 & 60.84 & 55.33 & 56.08 & 58.90 \\
\hline Signet Ring Cell Carcinoma & 137 & 179 & 222 & 218 & 243 & 284 & 11.75 & 12.44 & 13.67 & 13.60 & 14.01 & 12.07 \\
\hline Adenocarcinoma, Intestinaltype & 78 & 93 & 145 & 208 & 204 & 215 & 6.69 & 6.46 & 8.93 & 12.98 & 11.76 & 9.14 \\
\hline Carcinoma, Diffuse Type & 48 & 54 & 65 & 90 & 99 & 120 & 4.12 & 3.75 & 4.00 & 5.61 & 5.71 & 5.10 \\
\hline Carcinoma, Nos & 35 & 21 & 6 & 29 & 24 & 17 & 3.00 & 1.46 & 0.37 & 1.81 & 1.38 & 0.72 \\
\hline Mucinous Adenocarcinoma & 20 & 28 & 42 & 39 & 29 & 20 & 1.72 & 1.95 & 2.59 & 2.43 & 1.67 & 1.57 \\
\hline Mucin-Producing Adenocarcinoma & 30 & 25 & 23 & 22 & 31 & 30 & 2.57 & 1.74 & 1.42 & 1.37 & 1.79 & 1.23 \\
\hline \multicolumn{13}{|l|}{ Male } \\
\hline Aden & 2112 & 2586 & 2745 & 2648 & 2659 & 3322 & 68.39 & 68.59 & 65.17 & 61.60 & 59.30 & 61.54 \\
\hline Signet Ring Cell Carcinoma & 291 & 363 & 417 & 441 & 536 & 525 & 9.42 & 9.63 & 9.90 & 10.26 & 11.95 & 9.73 \\
\hline Adenocarcinoma, Intestinaltype & 210 & 321 & 456 & 551 & 625 & 571 & 6.80 & 8.51 & 10.83 & 12.82 & 13.94 & 10.58 \\
\hline Carcinoma, Diffuse Type & 90 & 127 & 167 & 175 & 169 & 263 & 2.91 & 3.37 & 3.96 & 4.07 & 3.77 & 4.87 \\
\hline Carcir & 85 & 41 & 12 & 81 & 41 & 40 & 2.75 & 1.09 & 0.28 & 1.88 & 0.91 & 0.74 \\
\hline Mucinous Adenocarcinor & 59 & 62 & 89 & 81 & 84 & 81 & 1.91 & 1.64 & 2.11 & 1.88 & 1.87 & 1.50 \\
\hline Mucin-Producing Adenocarcinoma & 67 & 70 & 86 & 70 & 70 & 72 & 2.17 & 1.86 & 2.04 & 1.63 & 1.56 & 1.33 \\
\hline
\end{tabular}

Table 3. Joinpoint Analyses of Cancers Percentage Allocated to the Seven Morphology Data for Stomach Cancer (2008-2003)

\begin{tabular}{|c|c|c|c|c|}
\hline & \multicolumn{2}{|c|}{ Female } & \multicolumn{2}{|c|}{ Male } \\
\hline & $\mathrm{APC}$ & $95 \% \mathrm{CI}$ & APC & $95 \% \mathrm{CI}$ \\
\hline Adenocarcinoma, Nos & -2.5 & -5.7 To 0.9 & $-2.9^{\wedge}$ & -4.8 To -1.0 \\
\hline Signet Ring Cell Carcinoma & 1.4 & -3.7 To 6.8 & 2.4 & -3.0 To 8.2 \\
\hline Adenocarcinoma, Intestinaltype & 11.3 & -4.3 To 29.3 & 11.7 & -1.3 To 26.4 \\
\hline Carcinoma, Diffuse Type & 7.9 & -1.0 To 17.7 & $8.8^{\wedge}$ & 2.7 To 15.2 \\
\hline Carcinoma, Nos & -15.1 & -48.3 То 39.6 & -13.8 & -50.3 To 49.6 \\
\hline Mucinous Adenocarcinoma & -2.8 & -16.2 Тo 12.9 & -2.6 & -10.3 To 5.7 \\
\hline Mucin-Producing Adenocarcinoma & -9.9 & -21.1 To 2.9 & $-8.7^{\wedge}$ & -13.0 To -4.3 \\
\hline
\end{tabular}

The percentage changes of assigned histology for men include ADENOCARCINOMA, NOS and MUCINPRODUCING ADENOCARCINOMA showed significant decline with annual percentage changes (APC) equal to - 2.87 and -8.74, respectively. Meanwhile CARCINOMA, DIFFUSE TYPE in men with APC $=8.76$ was significantly increased. Other trends about increase or decrease for histological studies showed no significant differences (Table 3).

\section{Discussion}

Stomach cancer is common among some parts of Iran and Central Asia (Somi et al., 2006). According to the results, the incidence of stomach cancer is on the rise among Iran. Babai et al. (2009) study, followed by Sajjadi (Sadjadi et al., 2003) show an increased trend for the incidence of stomach cancer in Ardabil . Somi et al. (2008), indicated an increase for incidence of stomach cancer among East Azerbaijan province and then Enayat (Najafi et al., 2011; Enayatrad and Salehiniya, 2014) confirmed same increased trend for incidence of stomach cancer throughout the country.

Najafi et al. (2011) confirmed the non-linear trend for stomach cancer increase in Iran. Aragons reported a reduction on stomach cancer cases from 1996 to 2004 in Spain, especially for non-cardia type. Nonham in the United Kingdom confirms the same results(Inoue and Tsugane, 2005). In the present study, the sex ratio (male to female) was equal to 2.54 .

Musav and Somi (2009) confirmed this sex ratio for stomach cancer which is consistent with England in 2005 which is a little more than USA, but it is significantly lower than Korea, Japan and Eastern Asia (Aghaei et al., 2013).

Aghaii et al. (2013) reported a sex ratio for stomach cancer equal to 1.99 which is consistent with country reports in 2007, 2006 and 2005 and is similar to our results. The World Health Organization reported a similar pattern of stomach cancer for both sexes, but the incidence is 2 times higher for men which confirm Aghaii findings (Haidari et al., 2012).

Our study results indicate that the most common type of stomach cancer in our country is ADENOCARCINOMA, NOS, with $62.50 \%$ of total cases and also increased trend for age standardized incidence rate (ASIR) for both sexes was observed . CARCINOMA DIFFUSE type is being increased in men but NOS, MUCIN-PRODUCING ADENOCARCINOMA had a reducing trend.

Heidar et al. (2012) confirms increases for incidence of cancer in both sexes Hajmanouchehri et al. (2013) reported ADENOCARCINOMA as the most common type of stomach cancer morphology, but an increase for diffuse / intestinal ratio was seen which is consistent with our study (Somi et al, 2006; Zhou et al., 2008; Babai et al., 2009; Mousavi et al., 2009; Rampazzo et al., 2012).

Abdirad et al. (2006) and Somi et al. (2006) confirm the increasing trend for adenocarcinoma for both sexes which is not consistent with Manuchehri and our results. 
Musavi reports a western pattern for adenocarcinoma increase among the country (Mousavi and Somi, 2009). In present study, CARCINOMA DIFFUSE type has an increasing trend in men. Rampazzo et al. (2012) also confirms increasing trend of CARCINOMA DIFFUSE type for both sexes and high incidence of disease among women over 40 years old. Schmassman et al. (2009) and Wu et al. (2009) found similar results similar to our study.

Noruzinia reported that the most common age of stomach cancer in Urmia district is attributed to old ones (over 70 years old) and reported adenocarcinoma as the most common type of stomach cancer with an incidence 3 times higher for men than women (Alireza et al., 2005). Parkin reported a reduction for incidence of adenocarcinoma in the last 50 years in the Western industrialized countries (Takiar et al., 2010).

According to data released by the Middle East Cancer Consortium (MECC), cancer incidence is low among 4 countries include Cyprus, Egypt, Arabian part of Israel and Jordan between 1996-2014, but it is high among Jews in Israel.

Stomach cancer is slightly higher in Iran than the MECC and USA and also has no significant difference with UK, but it is significantly lower than Japan, Korea and Southeast Asia (Aghaei et al., 2013). Differences in different countries' reports can be attributed to differences in morphologic classification of disease, reporting systems, population sample size and the diet.

Overall, the incidence of stomach cancer is increasing in Iran which and it can be seen among both sexes. Some part of this increase may be related to the establishment of cancer registration system in the country and also increasing risk factors (Alireza et al., 2005) and Helicobacter pylori infection (Kim, 2008).

Another study showed that the incidence of stomach cancer has not changed and still be recognized as a dominant disease among men. Also cardia and non-cardia distribution remained unchanged and we are witnessed only for changes in intestinal and diffuse type (Mousavi and Somi, 2009).

However, many studies have reported that the possible overall reduction in stomach cancer which possible reasons include diverse and rich diet, better storage methods for food stuffs including cooling methods and more efficient control over H. pylori infection. In our study, the reason that why incidence is higher among men is not clear, but one of the reasons maybe risky behaviors such as smoking among men, in addition to consumption of red and salty meat especially roasted meat.

Helicobacter pylori infection is the major risk factor for stomach cancer incidence. According to studies, incidence rate has been declined and further reduction among young people is expected. Other risk factors include the use of proton pump inhibitors, changes in the microbial environment of the stomach, loss the pylori colonization and lifestyle risk factors (smoking and diet).

Tumor location in the stomach, because of its important implications for treatment and prognosis is very important .Tumor in the middle third of the stomach has a better prognosis after surgical treatment while proximal tumors are more aggressive and have a poorer prognosis (Haidari et al., 2012). Several studies have reported an increased incidence of proximal tumors (Rampazzo et al., 2012).

Stomach cancer is not common in young people, but recent reports indicate that the incidence has been increased among Caucasian less than 40 years in the United States. Other studies have reported a high prevalence of diffuse type among young women and the high prevalence of intestinal type among older men (Shafaghi et al., 2012).

Performed studies in Iran are mostly related to the northern areas; especially northwest of the country and high prevalence of stomach cancer especially adenocarcinoma type is limited to these areas. It seems that these changes cannot be generalized to the whole country.

Also regarding studies about the pathology of stomach cancer, no subject has been discussed that either type of adenocarcinoma or carcinoma were increased or decreased.

Since the results of present study are based on data which obtained from the national Cancer data registry, no national study has found that to fully confirm or deny our results, so, extensive studies in all areas ranging from North, South, East, West and center of the country are needed among all age and ethnic groups to determine epidemiological aspects of the disease, in particular pathology and morphology of stomach cancer.

\section{References}

Abdi-Rad A, Ghaderi-Sohi S, Nadimi-Barfroosh H, et al (2006). Trend in incidence of gastric adenocarcinoma by tumor location from 1969-2004: a study in one referral center in Iran. Diagn Pathol, 1, 1-7.

Aghaei A, Ahmadi-Jouibari T, Baiki O, et al (2013). Estimation of the gastric cancer incidence in Tehran by two-source capture-recapture. Asian Pac J Cancer Prev, 14, 673-7.

Ahari SS, Agdam FB, Amani F, et al (2013). Analysis of the relationships between esophageal cancer cases and climatic factors using a geographic information system (GIS): a case study of Ardabil province in Iran. Asian Pac J Cancer Prev, 14, 2071-7.

Alireza S, Mehdi N, Ali M (2005). Cancer occurrence in Iran in 2002, an international perspective. Asian Pac J Cancer Prev, 6, 359.

Babaei M, Jaafarzadeh H, Sadjadi A, et al (2009). Cancer incidence and mortality in Ardabil: report of an ongoing population-based cancer registry in Iran, 2004-2006. Iran J Pub Health, 38, 35-45.

Babaei M, Mousavi S, Malek M, et al (2005). Cancer occurrence in Semnan province, Iran: results of a population-based cancer registry. Asian Pac J Cancer Prev, 6, 159-64.

Bener A, Ayub H, Kakil R, et al (2008). Patterns of cancer incidence among the population of Qatar: a worldwide comparative study. Asian Pac J Cancer Prev, 9, 19-24.

Derakhshan M, Yazdanbod A, Sadjadi A, et al (2004). High incidence of adenocarcinoma arising from the right side of the gastric cardia in NW Iran. Gut, 53, 1262-6.

Enayatrad M, Salehiniya H (2014). Trends in gastric cancer incidence in Iran. J Mazandaran Univers Med Sci (JMUMS), 24, 8-16.

Fritz AG (2000). International classification of diseases for oncology: ICD-O. 2000: World Health Organization.

Haidari M, Nikbakht MR, Pasdar Y, et al (2012). Trend analysis of gastric cancer incidence in Iran and its six geographical areas during 2000-2005. Asian Pac J Cancer Prev, 13, 
3335-41.

Hajmanoochehri F, Mohammadi N, Nasirian N, et al (2013). Patho-epidemiological features of esophageal and gastric cancers in an endemic region: a 20-year retrospective study. Asian Pac J Cancer Prev, 14, 3491-7.

Han MA, Choi KS, Lee H-Y, et al (2011). Current status of thyroid cancer screening in Korea: results from a nationwide interview survey. Asian Pac J Cancer Prev, 12, 1657-63.

Inoue M, Tsugane S (2005). Epidemiology of gastric cancer in Japan. Postgraduate Med J, 81, 419-24.

Kim J (2008). Protective effects of Asian dietary items on cancers-soy and ginseng. Asian Pac J Cancer Prev, 9, 543-8.

Moore MA, Eser S, Igisinov N, et al (2010). Cancer epidemiology and control in North-Western and Central Asia-past, present and future. Pancreas, 4, 7-1.9.

Mousavi SM, Gouya MM, Ramazani R, et al (2009). Cancer incidence and mortality in Iran. Ann Oncol, 20, 556-63.

Mousavi SM, Somi MH (2009). Gastric cancer in Iran 19662006. Asian Pac J Cancer Prev, 10, 407-12.

Najafi F, Mozaffari H, Karami M, et al (2011). Trends in incidence of gastrointestinal tract cancers in Western iran, 1993-2007. Iranian Red Crescent Med J, 13, 805.

Rampazzo A, Mott GL, Fontana K, et al (2012). Gastric adenocarcinoma trends in the central region of Rio Grande do Sul (Southern Brazil): what has changed in 25 years? Arquivos De Gastroenterologia, 49, 178-83.

Sadjadi A, Malekzadeh R, Derakhshan MH, et al (2003). Cancer occurrence in Ardabil: results of a population-based Cancer Registry from Iran. Intern J Cancer, 107, 113-8.

Schmassmann A, Oldendorf M-G, Gebbers J-O (2009). Changing incidence of gastric and oesophageal cancer subtypes in central Switzerland between 1982 and 2007. European J Epidemiol, 24, 603-9.

Shafaghi A, Mansour-Ghanaei F, Joukar F, et al (2012). Serum gastrin and the pepsinogen I/II ratio as markers for diagnosis of premalignant gastric lesions. Asian Pac J Cancer Prev, 14, 3931-6.

Somi MH, Farhang S, Mirinezhad SK, et al (2008). Cancer in East Azerbaijan, Iran: results of a population-based cancer registry. Asian Pac J Cancer Prev, 9, 327-30.

Somi MH, Mirinezhad K, Farhang S, et al (2006). Gastrointestinal cancer occurrence in East Azarbaijan: a five year study from North Western Iran. Asian Pac J Cancer Prev, 7, 309.

Takiar R, Nadayil D, Nandakumar A (2010). Projections of number of cancer cases in India (2010-2020) by cancer groups. Asian Pac J Cancer Prev, 11, 1045-9.

Wu H, Rusiecki JA, Zhu K, et al (2009). Stomach carcinoma incidence patterns in the United States by histologic type and anatomic site. Cancer Epidemiol Biomarkers Prev, 18, 1945-52.

Yazdizadeh B, Jarrahi AM, Mortazavi H, et al (2005). Time trends in the occurrence of major GI cancers in Iran. Asian Pac J Cancer Prev, 6, 130-4.

Zhou Y, Zhang Z, Zhang Z, et al (2008). A rising trend of gastric cardia cancer in Gansu Province of China. Cancer Letters, 269, 18-25. 